\title{
ON HEARING COLOURS - \\ CROSS-MODAL ASSOCIATIONS IN VOWEL PERCEPTION IN A NON-SYNAESTHETIC POPULATION
}

\author{
MAGDALENA WREMBEL \\ Adam Mickiewicz University, Poznań \\ magdala@aтu.edu.pl
}

\begin{abstract}
The present study is a continuation of previous investigations into the nature of sound-colour associations in a non-synaesthetic population conducted on English and Polish vowel sound systems and it aims at providing further evidence for the non-arbitrary nature of cross-modal mappings. The experiment ${ }^{1}$ was run on a specially designed computer program and involved 90 participants who were asked to match randomised auditory stimuli (12 English vowel sounds recorded in 2 conditions: in isolation and in a CVC context) with one of 11 basic colours (red, yellow, green, blue, brown, purple, pink, orange, black, white and grey) presented as coloured rectangles on a computer screen. The program kept record of the colour choice and reaction time of the participants, who fell into 2 groups with respect to the level of their language proficiency and phonetic awareness.

An analysis of the results revealed statistically significant interactions between specific colours and individual vowel sounds for all 12 English vowels examined in the combined analysis; for 10 vowels in Condition 1 (isolated auditory stimuli) and for 7 vowels in Condition 2 (stimuli in the CVC context). A group effect was not found to be significant as far as the quality of mappings was concerned; however, in the case of reaction times the less advanced learners took significantly longer to assign colours to sounds in context. The findings indicate that vowel-sound mappings in non-synaesthetic perception appear non-arbitrary and follow the general tendencies in which bright colours (yellow, green) are associated with high front vowel sounds, whereas dark colours (brown, blue, black) are attributed to back vowels, while open sounds tend to be perceived as red and central vowels are mapped onto achromatic grey.
\end{abstract}

KEYWORDS: Sound-colour mappings; vowel colour; synaesthesia.

\section{Introduction}

The present contribution promotes a non-traditional approach to the study of speech perception mediated through colour with a view to facilitating the process of second

\footnotetext{
${ }^{1}$ I wish to express my thanks to Karolina Rataj for her assistance in designing the research.
} 
language phonological acquisition. It relies on the concept of the non-modularity of human perception and common occurrence of cross-modal audio-visual mechanisms in the non-synaesthetic population (cf. Calvert 2001).

The study investigates the nature of cross-modal mappings between vowel sound stimuli and colours based on the assumptions stemming from research on vowel colour, synaesthesia, and sound symbolism. It also pertains to hypotheses put forward by various researchers including Pythagoras and Sir Isaac Newton, about the existence of a physical relationship between the frequencies of light and sound that are responsible for the sub-modality of colour and pitch (cf. Lyons 2001).

\section{Theoretical assumptions}

\subsection{Vowel colour}

The application of colour terminology with relation to vowels can be traced back to Jakobson (1962), who pointed to the regularity of colour associations in coloured hearing synaesthesia - identifying close connections of the vowels /o/ and / $\mathrm{u} /$ with darker colours, /e/ and /i/ with brighter colours, and /a/ with red. Jakobson claimed that "there seems to be a phenomenal affinity between optimal chromaticity (pure red) and vocalic compactness, [between] attenuated chromaticity (yellow-blue) and vocalic diffuseness, optimal achromaticity (black-white) and consonantal diffuseness, attenuated achromaticity (greyed) and consonantal compactness; and, finally, between the value axis of colour (dark-light) and the tonality axis in language" (Jakobson 1962: 488, footnote). Jakobson called for research into synaesthetic associations between phonemic features and colour attributes in order to provide insights into the perceptual aspects of speech sounds.

The term "vowel colour" was also used by Donegan (1985) to refer to such features as palatality and labiality. Donegan stated that palatal vowels, traditionally referred to as front vowels, with high F2 and a considerable distance between F1 and F2, tend to be perceived as "bright" as opposed to "dark" labial vowels which are characterised by low F2 and a small distance between F1 and F2. Vowels which are neither palatal nor labial are called plain or achromatic (Donegan 1985: 66-67). Although labiality and palatality are considered the most basic, i.e. primary colours, Donegan admitted that the notion of vowel colour requires further examination.

Furthermore, Klunder (2003) referred to vowel colour when investigating the socalled "auditory colour constancy", i.e. the auditory equivalent of visual colour constancy that people display when listening to speech sounds.

Very few studies to date have explored this synaesthetic-like phenomenon of making associations between colours and auditory stimuli (whether pure tones or vowel sounds). Flagg and Stewart (1985), on the basis of their research into consonantal perception by using 6 primary colours, concluded that colour can indeed be used to study 
speech perception, while Dailey et al. (1997) investigated the relation between creativity, synaesthetic tendencies and physiognomic perception measured by rating colours using adjectives with emotional connotations. Their findings demonstrated that creative individuals have access to a primary process thinking - that assumes a unity of different sensory modalities, since they exhibited stronger associations between colours and vowels, as well as tones and emotional terms.

One of the few experimental studies conducted on a non-synaesthetic correspondence between colours and vowels is that of Miyahara et al. (2006). In a perceptual similarity experiment, they examined the patterns of correspondences between 4 selected colours (red, blue, yellow and green) and 5 recorded vowels (/a/, /e/, /i/, /o/, /u/). The auditory stimuli included a normal female and a male voice as well as manipulated stimuli (i.e. high-pitch male voice and low-pitch female voice). The Japanese subjects were asked to make choices between a pair of colours presented as coloured rectangles on a computer screen simultaneously with a randomised auditory stimulus. The findings revealed clear regularities; red was chosen for /a/ significantly more than any other vowel, yellow was chosen for /i/ significantly more than any other vowel, blue was chosen for /o/ significantly more than any other vowel and for /e/ significantly more than for $/ \mathrm{i} /$ and $/ \mathrm{a} /$. There was also an effect of gender and pitch on colour choice; the analysis revealed a significant interaction between the red choice for /a/ and normal female voice, blue choice for /o/ and normal male voice, as well as yellow choice for $/ \mathrm{i} /$ and normal female voice and high-pitch male voice. Furthermore, Miyahara et al. (2006) compared their results to patterns of vowel-colour mappings for the synaesthetic population extracted from Day's (2004) study. As a result, they identified similar patterns of correspondence in both types of populations (referred to as strong and weak synaesthesia), with the ratios of colour-vowel choices being slightly lower for the nonsynaesthetic group.

In an attempt to account for the correspondence observed across languages and types of synaesthesia, Miyahara et al. (2006) pointed to the contrast between front and back vowels as related to the yellow-blue contrast in the colour spectrum, with some indications concerning the role of formant structures. A similar hypothesis was earlier put forward by Slawson (1985), according to whom the sound-colour mapping is a function of the frequencies of the first two resonances, which organise sound features. However, an unequivocal explanation for the observed patterns of sound-colour correspondence still remains to be provided.

\subsection{Research on synaesthesia}

The relationships between sensory sub-modalities have been most extensively explored in studies on synaesthesia, a condition which involves involuntary physical experience of cross-sensory associations, particularly between sounds (mainly vowels) and colour. It concerns subjective sensation of a sense that is different from the one being stimu- 
lated. Synaesthesia has been confirmed by neuro-imaging data (PET) which report different cortical blood flow patterns compared to the normal population (Harrison 2001).

Although different forms of synaesthesia trigger vivid cross-modal idiosyncratic associations, the sound-colour mappings tend to demonstrate fairly non-arbitrary patterns. Synaesthetic research reports a strong correlation between auditory pitch and visual luminance, as well as a general tendency to associate high pitch sounds with light colours and low tones with darker hues (cf. e.g. Hubbard 1996; Simner et al. 2005; Ward et al. 2006). Some of the existing theories addressing the causes of synaesthesia point to the fact that strong tendencies in inter-sensory, cross-modal matching especially between auditory and visual stimuli have also been found in people without synaesthesia (Marks 1975; Harrison 2001).

Ward et al. (2006: 264) suggest that research on synaesthesia can be used "to inform theories of normal cognition" since people who report this condition are hypothesised to recruit analogous mechanisms to the ones used in non-synaesthetic cross-modal perception. The differences lie in the nature of these sound-colour mappings; in the general population, they are strategic rather than automatic and are not accompanied by an explicit perceptual experience.

Moreover, a concept of weak synaesthesia was proposed by Martino and Marks (2001), according to whom this synaesthesia is characterised by cross-sensory correspondences expressed through language, perceptual similarity, and perceptual interactions during information processing. Unlike strong cross-modal associations of synaesthetes, this weak form can be experienced by the non-synaesthetic population.

However, very few studies to date have explored the phenomenon of making associations between the colour spectrum and speech sounds in non-synaesthetic perception (cf. Flagg and Stewart 1985; Dailey et al. 1997; Miyahara et al. 2006) and, therefore, the present author's series of investigations are intended to further examine the potential cross-sensory correspondence for speech.

\subsection{Sound symbolism}

Synaesthetic-like inter-sensory associations of sounds and colours may also be interpreted as manifestations of phonetic symbolism, i.e. non-arbitrary representations of a phoneme by specific semantic criteria. The term is used interchangeably with iconicity, to denote sound symbolic relationships which closely link semantic interpretation with phonological representation (Hinton et al. 1994). Sound symbolism is concerned with cognitive rather than lexical or linguistic properties of phonemes and it is usually manifested in the matching of a phoneme to polar adjective pairs like small/big, bright/dark, etc.

Different manifestations of sound symbolism have been identified including synaesthetic and phonesthetic associations. Phonesthetics is concerned with the aesthetic symbolism of sounds, for example, /ii/ and/I/ represent high-frequency sounds and are typi- 
cally associated with small size, sharpness, rapid movement, and physically high objects. On the other hand, $/ \mathrm{u} /$ and $/ \mathrm{a} /$ represent low frequency sounds and tend to symbolise large size, softness and slow movement, and physically low objects (Hinton et al. 1994). Day (2004) claims that trends in synaesthetically coloured phonemes seem to resemble worldwide tendencies in sound symbolism.

\section{Experiment on colour-sound associations}

The present experiment was intended as a follow-up to two previous investigations into the nature of sound-colour associations in a non-synaesthetic population in Polish vowel sounds (Wrembel 2007) and English vowel sounds (Wrembel and Rataj 2008).

Its specific aim was to provide further evidence for the non-arbitrary nature of cross-modal mappings by a partial replication of the second study on English pure vowels with a number of adaptations, including (1) a doubled number of new participants, (2) two groups of different proficiency levels and different degrees of established mental representations of the English sound system, (3) two conditions of presentation of auditory stimuli.

The research questions that were addressed in the study were as follows:

- Are English vowel sounds mapped on the visual domain of the colour spectrum in a consistent and non-arbitrary manner?

- To what extent are sound-colour associations conditioned by the participants' level of proficiency and previous phonetic training in the English sound system?

- Does the condition of presentation of auditory stimuli have any impact on the crossmodal mappings?

\subsection{Method}

\subsubsection{Participants}

The participants consisted of 90 Polish students of English at Adam Mickiewicz University in Poznań (69 females and 21 males, whose mean age was 20.5). They differed in terms of the level of their proficiency in English: Group 1 involved 1st year students with intermediate proficiency and little previous phonetic training, thus any mental representations of the English vowel repertoire were virtually nonexistent, whereas Group 2 included 3rd year students with advanced proficiency in English who had been subject to intensive phonetic training in the course of their studies, thus their mental representation of the English sound system was well established and the degree of their metalinguistic awareness was high. At the time of the experiment they were ignorant about the phenomenon of synaesthesia and did not report any synaesthetic experiences themselves. 


\subsubsection{Materials}

The sound stimuli used in the experiment included 12 English pure vowel sounds recorded in isolation and in a CVC context (i.e., b_d). The stimuli were recorded by a male native speaker of English in a professional recording studio as 16-bit mono files at a sampling frequency of $16000 \mathrm{~Hz}$ using the Audacity software.

\subsubsection{Procedure}

The design of the experiment replicated closely the one applied in the previous experiments (cf. Wrembel 2007; Wrembel and Rataj 2008) to allow for reliable comparisons. The experiment was run on a specially designed computer program, implemented in Visual Basic, that offered the following functionality:

- it played a given (parameterised) number of sounds - in every testing cycle the order of the sounds was randomised;

- it displayed the palette of 11 basic colours - in every testing cycle the position of every colour was randomised;

- it registered in a text file the sound that was played, the selected colour, tester's name, and response time.

The experiment was carried out as a series of individual sessions. The participants were seated in front of a computer screen in a dark room. They were instructed to listen to individual sounds and choose one colour from a palette of 11 basic colours after a given sound had been played. The colours could be chosen from a palette of 11 basic colours as specified by Berlin and Kay (1969), including red, yellow, green, blue, brown, purple, pink, orange, black, white and grey. The colours were selected from over 500 samples available at $<\mathrm{http}: / /$ cloford.com $/$ resources $/$ colours $/ 500 \mathrm{col}$.htm $>$ (a page listing over 500 colours by colour name, Hex value, RGB value and Microsoft Access code number). Each colour could be represented numerically in the RGB (red, green, blue) space, with values ranging from 0 to 255 (where 0, 0, 0 stands for black and 255, 255, 255 represents white). Previous studies of this type carried out on synaesthete populations used the Munsell colour space or Windows API Choose Colour dialogue box (cf. Ward et al. 2006). The present study, however, used a limited selection of the colour spectrum to simplify the experiment by concentrating only on focal universal colours and to avoid a source of unnecessary noise in the data. It was assumed that a non-synaesthetic population would not require such fine-grained colour specifications as it does not report, unlike synaesthetes, any specific colour visual experiences.

The test was self-paced, i.e. the participants could play a given sound as many times as they wished, but once a colour was selected they were not able to modify it. The participant's choice was made by clicking on one of 11 coloured rectangles presented in 3 
rows against a light grey background. The colour palette appeared automatically on the screen with a 2 second delay after a "Play" button was clicked in order to play a particular vowel sound. The placement of the coloured rectangles on the screen was randomised to avoid any fixed location in space.

The auditory stimuli were presented in two conditions: first the test was run on 12 English vowel sounds recorded in isolation, the second part of the experiment involved the same vowel sounds presented in a CVC context (b_d). Consequently, each participant listened to 24 stimuli in order to check for the consistency of sound-colour associations and to investigate the potential influence of the context on the choices made. In total, the experiment generated 2,160 tokens of cross-modal mappings that were all subject to further analysis.

\subsection{Results}

\subsubsection{Sound-colour mappings}

A statistical analysis was performed by means of SPSS in order to examine whether the mappings between the English vowel sounds and the eleven basic colours were random or not. The results of a non-parametric chi-square test point to the statistical significance of sound-colour interactions for all vowel sounds under investigation in both conditions and for both groups of participants analysed jointly ( $<<.05$, see Table 1$)$.

Table 1. Pearson chi-square analysis of vowel and colour interactionin combined conditions.

\begin{tabular}{lcccccccccccc}
\hline Vowel & $æ$ & o: & $\Lambda$ & $\mathrm{a}:$ & $\mathrm{e}$ & $3:$ & $\mathrm{I}$ & $\mathrm{i}:$ & $\mathrm{p}$ & $\partial$ & $\mathrm{U}$ & $\mathrm{u}$ : \\
\hline$\chi^{2}$ & 36 & 36 & 23 & 64 & 20 & 63 & 26 & 96 & 24 & 49 & 40 & 41 \\
$\mathrm{df}$ & 10 & 10 & 10 & 10 & 10 & 10 & 10 & 10 & 10 & 10 & 10 & 10 \\
$\mathrm{p}$ & .00 & .00 & .01 & .00 & .03 & .00 & .00 & .00 & .01 & .00 & .00 & .00 \\
\hline
\end{tabular}

Specific colour associations for the English vowel sounds in both conditions are presented in Table 2 (overleaf). The percentage values reflect the strength of sound-colour mappings. Highlighted values indicate the colours that were assigned to particular vowels at an above-chance level, as demonstrated by the chi-square test results comparing the observed values to the expected ones.

The results were analysed also for both conditions separately. In Condition 1, when auditory stimuli were presented as individual sounds in isolation, the analysis revealed that the sound vs. colour interaction was significant for 10 out of 12 of the English vowels under investigation ( $\mathrm{p}<.05$, see Table 3 , overleaf). The mappings appeared randomly dispersed across the colour spectrum only for two vowels, i.e. $/ \Lambda /$ and $/ \mathrm{e} /$, 
Table 2. Colour associations for English vowels - combined analysis.

\begin{tabular}{|c|c|c|c|c|c|c|c|c|c|c|c|c|}
\hline & $\Lambda$ & $\mathrm{a}:$ & $æ$ & e & 3: & I & i: & $\mathrm{p}$ & 0: & $\partial$ & $U$ & u: \\
\hline brown & $14 \%$ & $16 \%$ & $12 \%$ & $10 \%$ & $18 \%$ & $7 \%$ & $4 \%$ & $11 \%$ & $18 \%$ & $9 \%$ & $10 \%$ & $19 \%$ \\
\hline blue & $10 \%$ & $13 \%$ & $5 \%$ & $13 \%$ & $14 \%$ & $10 \%$ & $12 \%$ & $8 \%$ & $15 \%$ & $8 \%$ & $8 \%$ & $15 \%$ \\
\hline red & $11 \%$ & $20 \%$ & $18 \%$ & $12 \%$ & $6 \%$ & $9 \%$ & $10 \%$ & $6 \%$ & $9 \%$ & $8 \%$ & $6 \%$ & $8 \%$ \\
\hline orange & $8 \%$ & $8 \%$ & $12 \%$ & $8 \%$ & $4 \%$ & $12 \%$ & $8 \%$ & $17 \%$ & $7 \%$ & $9 \%$ & $12 \%$ & $11 \%$ \\
\hline yellow & $5 \%$ & $12 \%$ & $11 \%$ & $8 \%$ & $3 \%$ & $13 \%$ & $26 \%$ & $12 \%$ & $7 \%$ & $8 \%$ & $3 \%$ & $5 \%$ \\
\hline green & $8 \%$ & $9 \%$ & $10 \%$ & $14 \%$ & $9 \%$ & $12 \%$ & $16 \%$ & $9 \%$ & $3 \%$ & $6 \%$ & $7 \%$ & $3 \%$ \\
\hline grey & $10 \%$ & $2 \%$ & $3 \%$ & $9 \%$ & $15 \%$ & $14 \%$ & $3 \%$ & $9 \%$ & $8 \%$ & $22 \%$ & $18 \%$ & $7 \%$ \\
\hline purple & $14 \%$ & $4 \%$ & $5 \%$ & $4 \%$ & $9 \%$ & $7 \%$ & $5 \%$ & $6 \%$ & $7 \%$ & $7 \%$ & $13 \%$ & $8 \%$ \\
\hline black & $3 \%$ & $6 \%$ & $10 \%$ & $8 \%$ & $16 \%$ & $4 \%$ & $2 \%$ & $4 \%$ & $12 \%$ & $4 \%$ & $12 \%$ & $10 \%$ \\
\hline white & $10 \%$ & $4 \%$ & $8 \%$ & $4 \%$ & $2 \%$ & $3 \%$ & $5 \%$ & $10 \%$ & $9 \%$ & $13 \%$ & $3 \%$ & $7 \%$ \\
\hline pink & $5 \%$ & $7 \%$ & $5 \%$ & $8 \%$ & $4 \%$ & $8 \%$ & $7 \%$ & $7 \%$ & $5 \%$ & $4 \%$ & $9 \%$ & $8 \%$ \\
\hline
\end{tabular}

Table 3. Colour associations for English vowels in isolation.

\begin{tabular}{|c|c|c|c|c|c|c|c|c|c|c|c|c|}
\hline & $\Lambda$ & a: & $æ$ & $\mathrm{e}$ & 3: & I & i: & $\mathrm{p}$ & ๑: & $\partial$ & $U$ & u: \\
\hline brown & $14 \%$ & $18 \%$ & $12 \%$ & $12 \%$ & $17 \%$ & $6 \%$ & $0 \%$ & $11 \%$ & $24 \%$ & $6 \%$ & $10 \%$ & $22 \%$ \\
\hline blue & $8 \%$ & $12 \%$ & $7 \%$ & $16 \%$ & $20 \%$ & $16 \%$ & $12 \%$ & $10 \%$ & $13 \%$ & $7 \%$ & $7 \%$ & $18 \%$ \\
\hline red & $14 \%$ & $20 \%$ & $20 \%$ & $13 \%$ & $10 \%$ & $11 \%$ & $12 \%$ & $6 \%$ & $9 \%$ & $10 \%$ & $8 \%$ & $7 \%$ \\
\hline orange & $7 \%$ & $7 \%$ & $11 \%$ & $3 \%$ & $2 \%$ & $12 \%$ & $10 \%$ & $21 \%$ & $10 \%$ & $8 \%$ & $16 \%$ & $8 \%$ \\
\hline yellow & $6 \%$ & $11 \%$ & $7 \%$ & $10 \%$ & $4 \%$ & $9 \%$ & $34 \%$ & $11 \%$ & $7 \%$ & $8 \%$ & $4 \%$ & $2 \%$ \\
\hline green & $8 \%$ & $10 \%$ & $10 \%$ & $13 \%$ & $11 \%$ & $9 \%$ & $17 \%$ & $8 \%$ & $2 \%$ & $4 \%$ & $9 \%$ & $4 \%$ \\
\hline grey & $8 \%$ & $1 \%$ & $4 \%$ & $8 \%$ & $8 \%$ & $13 \%$ & $0 \%$ & $9 \%$ & $7 \%$ & $27 \%$ & $14 \%$ & $6 \%$ \\
\hline purple & $14 \%$ & $3 \%$ & $6 \%$ & $4 \%$ & $8 \%$ & $6 \%$ & $3 \%$ & $7 \%$ & $4 \%$ & $7 \%$ & $16 \%$ & $9 \%$ \\
\hline black & $3 \%$ & $7 \%$ & $16 \%$ & $9 \%$ & $13 \%$ & $4 \%$ & $1 \%$ & $4 \%$ & $9 \%$ & $3 \%$ & $4 \%$ & $11 \%$ \\
\hline white & $10 \%$ & $3 \%$ & $4 \%$ & $4 \%$ & $3 \%$ & $4 \%$ & $4 \%$ & $9 \%$ & $8 \%$ & $17 \%$ & $1 \%$ & $9 \%$ \\
\hline pink & $8 \%$ & $8 \%$ & $3 \%$ & $7 \%$ & $3 \%$ & $10 \%$ & $6 \%$ & $4 \%$ & $7 \%$ & $4 \%$ & $11 \%$ & $4 \%$ \\
\hline
\end{tabular}

whereas for the remaining vowels in Condition 1, they demonstrated clear colour preferences and non-random distribution.

Table 3 presents the actual values for specific colour associations for English vowels in isolation, with highlighted strongest mappings significant at $p<.05$ level.

As far as Condition 2 is concerned (i.e., vowel stimuli presented in the CVC context) the chi-square results point to a statistical significance of non-random colour assignment for 7 out of 12 of the investigated vowels $(\mathrm{p}<.05)$. The vowels that demonstrated random distribution of colours included $/ \Lambda /, / \mathrm{e} /, / \mathrm{p} /, / \mathrm{a} /$ and $/ \mathrm{u} / /$. The respective 
Table 4. Colour associations for English vowels in context.

\begin{tabular}{lrrrrrrrrrrrr}
\hline & $\Lambda$ & a: & \multicolumn{1}{c}{$æ$} & \multicolumn{1}{c}{ e } & \multicolumn{1}{c}{3 : } & \multicolumn{1}{c}{ I } & i: & p & 0: & ə & U & u: \\
\hline brown & $13 \%$ & $14 \%$ & $11 \%$ & $9 \%$ & $18 \%$ & $9 \%$ & $8 \%$ & $11 \%$ & $11 \%$ & $13 \%$ & $10 \%$ & $15 \%$ \\
blue & $12 \%$ & $14 \%$ & $4 \%$ & $11 \%$ & $8 \%$ & $5 \%$ & $11 \%$ & $5 \%$ & $17 \%$ & $10 \%$ & $10 \%$ & $13 \%$ \\
red & $8 \%$ & $21 \%$ & $16 \%$ & $10 \%$ & $2 \%$ & $8 \%$ & $9 \%$ & $7 \%$ & $10 \%$ & $7 \%$ & $4 \%$ & $9 \%$ \\
orange & $10 \%$ & $9 \%$ & $12 \%$ & $13 \%$ & $5 \%$ & $12 \%$ & $7 \%$ & $13 \%$ & $4 \%$ & $11 \%$ & $8 \%$ & $14 \%$ \\
yellow & $5 \%$ & $12 \%$ & $15 \%$ & $5 \%$ & $2 \%$ & $16 \%$ & $17 \%$ & $12 \%$ & $8 \%$ & $9 \%$ & $1 \%$ & $8 \%$ \\
green & $9 \%$ & $8 \%$ & $10 \%$ & $15 \%$ & $8 \%$ & $15 \%$ & $16 \%$ & $11 \%$ & $4 \%$ & $8 \%$ & $5 \%$ & $2 \%$ \\
grey & $12 \%$ & $2 \%$ & $1 \%$ & $11 \%$ & $22 \%$ & $15 \%$ & $7 \%$ & $10 \%$ & $9 \%$ & $17 \%$ & $21 \%$ & $8 \%$ \\
purple & $14 \%$ & $4 \%$ & $5 \%$ & $4 \%$ & $10 \%$ & $9 \%$ & $8 \%$ & $5 \%$ & $10 \%$ & $7 \%$ & $11 \%$ & $8 \%$ \\
black & $3 \%$ & $5 \%$ & $5 \%$ & $8 \%$ & $18 \%$ & $3 \%$ & $3 \%$ & $4 \%$ & $14 \%$ & $5 \%$ & $20 \%$ & $9 \%$ \\
white & $11 \%$ & $5 \%$ & $12 \%$ & $4 \%$ & $1 \%$ & $2 \%$ & $7 \%$ & $12 \%$ & $10 \%$ & $10 \%$ & $4 \%$ & $4 \%$ \\
pink & $3 \%$ & $5 \%$ & $8 \%$ & $10 \%$ & $5 \%$ & $5 \%$ & $9 \%$ & $10 \%$ & $3 \%$ & $4 \%$ & $7 \%$ & $11 \%$ \\
\hline
\end{tabular}

strengths of sound-colour mappings for vowels presented in context are demonstrated in Table 4 with highlighted values indicating non-random colour assignments for particular sounds.

As can be seen from Tables 2-4, the distribution patterns of vowel-sound mappings demonstrate comparable tendencies for both conditions and for the combined analysis (i.e., average results). On the whole, the strongest mappings were found for /i:/ in all conditions, as it triggered significant associations with yellow (34\% in isolation; $26 \%$ on average) and green (17\% in isolation, $16 \%$ on average). Furthermore, /a:/ was consistently mapped onto red $(20 \%)^{2}$ and brown $(16 \%)$, whilst /æ/ was mapped onto red $(18 \%)$ and orange (12\%). Colour distribution patterns for $/ \mathrm{a} /$ point to a strong correlation with grey ( $27 \%$ in isolation, $22 \%$ on average), whereas $/ 3: /$ yielded a mixture of associations with grey ( $22 \%$ in context, $15 \%$ on average), brown (18\%), and black (16\%). The vowel /u:/ demonstrated high non-random associations in isolation but not in context, with brown ( $22 \%$ in isolation, $19 \%$ on average) and blue (18\% in isolation, $15 \%$ in context). Similarly, $/ \mathrm{\jmath}:$ generated strong mappings with brown ( $24 \%$ in isolation, $18 \%$ on average). The strength of the remaining mappings was at a moderate level, with /I/ generating a mixture of grey, green and yellow (ca. 13\%); / $\mathrm{p} /$ - orange $(17 \%)$ and yellow (12\%); and / / grey (18\%) and purple (13\%). The vowels that yielded weak, nonrandom patterns of sound-colour distributions only in the combined analysis of the two conditions were $/ \Lambda /$ and $/ \mathrm{e} /$, the former triggering associations mostly with brown $(14 \%$ on average) and purple (14\% on average); and the latter with green (14\% on average) and blue (13\% on average).

\footnotetext{
${ }^{2}$ In those cases when only one percentage value is provided, it refers to the average strength of the vowelcolour mapping generated in the combined condition analysis.
} 
It was found that if a particular sound was associated above chance level with more than one colour, then these pairs of colours were usually closely related on the colour spectrum, e.g. yellow and green for $/ \mathrm{i}$ /, blue and green for $/ \mathrm{e} /$, red and orange for $/ \mathfrak{æ} /$, grey and black for $/ 3: /$.

Figure 1 offers a visual presentation of the multi-dimensional correspondence analysis performed on the associations between the English vowel sounds in both conditions and the selected 11 basic colours. Peripheral locations of sounds and colours indicate their strong mutual interaction, whereas the most central positioning points to much weaker specific mappings (i.e. vowels close to the axis intersection interacted with various colours to a comparable degree). Furthermore, the sounds were located on the same side of an axis and in proximity of the colours that they were most frequently associated with, whereas the colours located on the opposite side of the axis from a particular sound did not yield any non-random distribution patterns for that sound.

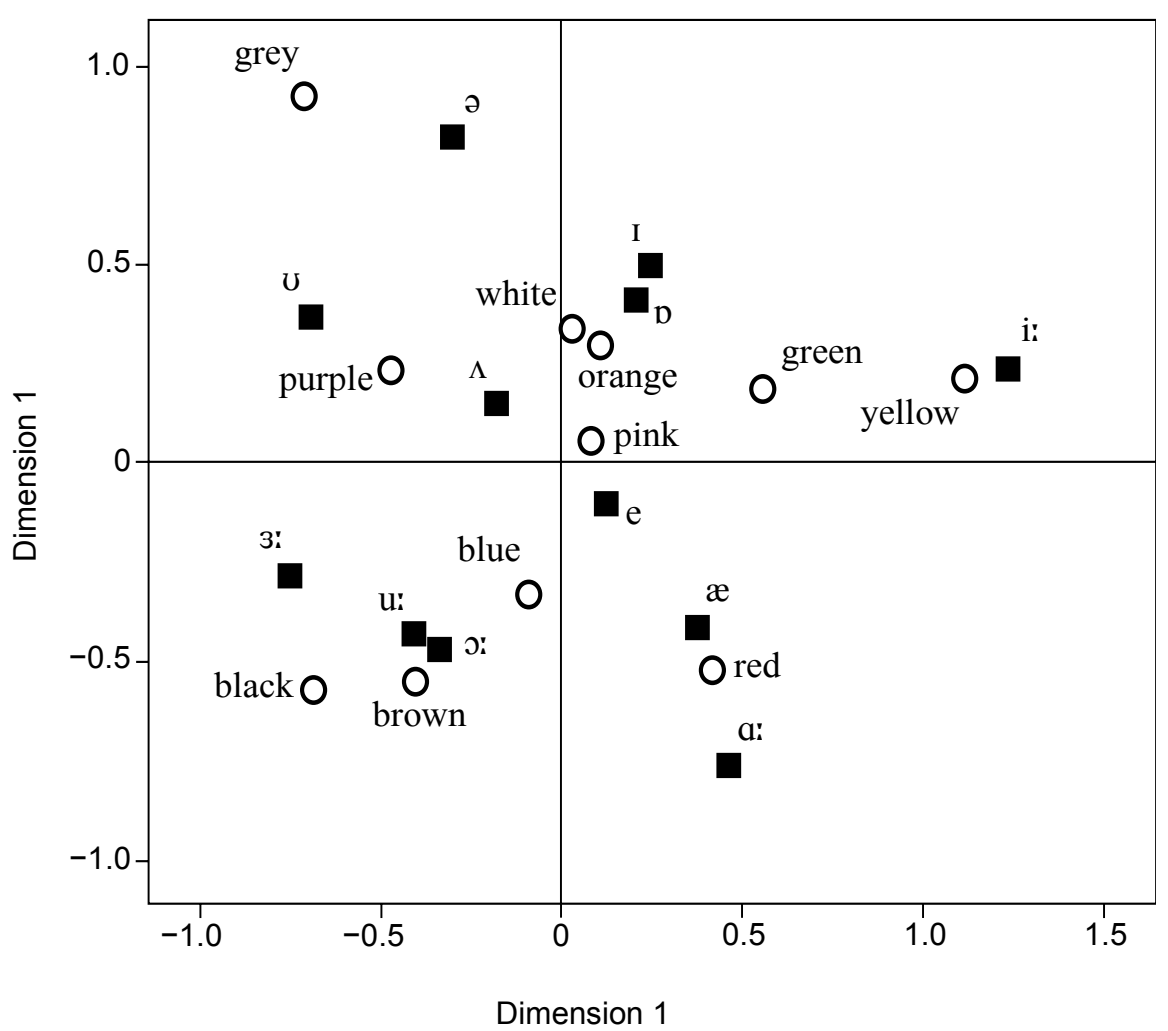

Figure 1. Multidimensional correspondence analysis for English vowel-colour associations. 


\subsubsection{Colour and vowel categories}

Further analysis of sound-colour mappings accounted for vowel categories (high/ central/back and high/mid/low) rather than individual sounds (as in 3.2.1). The results of a chi-square test indicated that the assignment of colours was non-random for all the 6 categories $(p<.01)$. As it was found when $\mathrm{c}$ omparing the observed values to the expected ones, the non-random patterns of distributions pointed to yellow, green and red for the front vowels; grey and brown for central; and brown and black for the back vowels. In the case of high vowels, the most frequently assigned colours were yellow and blue; for mid vowels - grey and brown; and for low ones - red, brown and orange (see Table 5).

Table 5. Colour interactions for vowel categories (presented for relevant colours only).

\begin{tabular}{lcccccc}
\hline & Front & Central & Back & High & Mid & Low \\
\hline yellow & $14 \%$ & & & $12 \%$ & & \\
green & $13 \%$ & & & & & $11 \%$ \\
red & $12 \%$ & & & & $14 \%$ & \\
grey & & $16 \%$ & & & $14 \%$ & $13 \%$ \\
brown & & $14 \%$ & $15 \%$ & & & \\
blue & & & $12 \%$ & $12 \%$ & & \\
\hline
\end{tabular}

\subsubsection{Group comparison}

In order to address the second research question on the potential conditioning effect of the level of proficiency and the existence of any previous phonetic training, a group comparison was performed by means of a loglinear analysis. The partial association analysis revealed no statistically significant interactions between colour/sound assignment and group, the only significant interactions being between colours and sounds $(\mathrm{p}<.01)$ - see Table 6.

Table 6. Partial associations analysis for group/colour/sound interactions.

\begin{tabular}{lrrrc}
\hline & df & Partial $\chi^{2}$ & Sig. & No. of iterations \\
\hline color1*group & 121 & 423.962 & 0.000 & 2 \\
color1*year & 22 & 10.155 & 0.985 & 2 \\
sound*group & 22 & 0.151 & 1.000 & 2 \\
color1 & 11 & 460.262 & 0.000 & 2 \\
sound & 11 & 0.000 & 1.000 & 2 \\
group & 2 & 1784.009 & 0.000 & 2 \\
\hline
\end{tabular}


However, when the reaction times (measured as the time that elapsed from playing the sound for the first time and clicking on a particular colour rectangle) of both groups were compared by means of a T-test, it was found that less advanced learners (Group 1) take significantly longer to assign colours to sounds in context $(\mathrm{t}=3.2, \mathrm{p}<.01)$ than phonetically trained learners (Group 2) - see Figure 2.

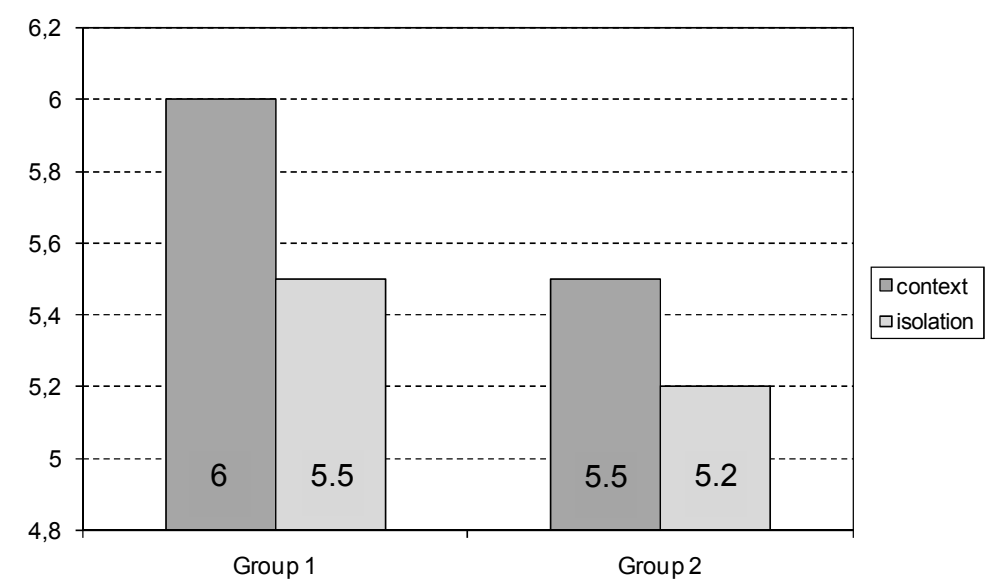

Figure 2. Mean reaction time (in seconds) for Group 1 and Group 2 in two different conditions.

\subsubsection{Condition comparison}

The impact of the condition of presentation of the auditory stimulus (i.e. vowels in isolation vs. the CVC context) was analysed in terms of the quality of sound-colour mappings and in terms of reaction times. The quality of sound-colour mappings in the two conditions exhibited some differences that are presented in Tables 2 and 3; however, the general pattern of distribution of the most significant sound-colour interactions remains highly comparable (see 3.2.1). The consistency of participants' choices between Condition 1 and Condition 2 was calculated, the value of the Kappa measure of agreement being 0.13 , which indicates a weak agreement. The overall consistency of individual vowel-colour assignments in Condition 1 vs. Condition 2 was $22 \%$.

As far as the reaction times in the two different conditions are concerned, the results of the T-test point to significantly longer responses to vowels presented in context than to vowels presented in isolation $(\mathrm{t}=5.4, \mathrm{p}<.01)$ when the vowels are analysed jointly. However, when individual vowels are subject to the analysis, significantly longer reaction times $(\mathrm{p}<.01)$ were observed in the context condition for the following 5 vowels: $/ \mathfrak{x} /, / \mathrm{I} /, / \mathrm{ii} /, / \mathrm{\jmath} /$ and $/ \mathrm{e} /($ see Fig. 3). 


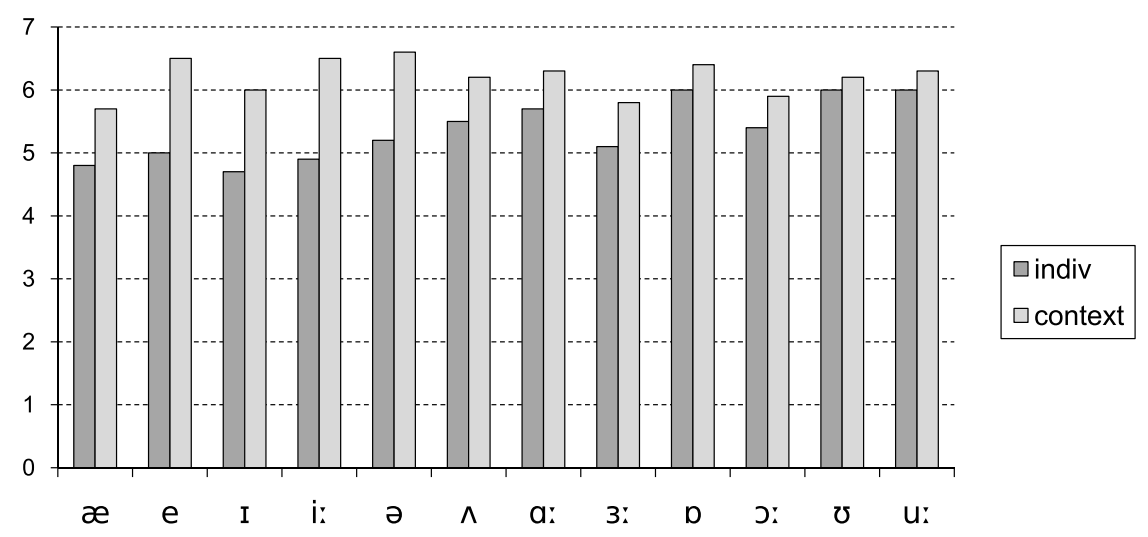

Figure 3. Mean reaction times (in seconds) for vowels in the two conditions (in isolation vs. in context).

\subsubsection{Consistency with previous studies}

A comparison with the results of the previous studies (Wrembel 2007; Wrembel and Rataj 2008) demonstrates that the associations with specific colours evoked by the perception of L2 speech sounds follow very similar patterns of distribution across the studies (see Table 7). Moreover, L2 sound-colour mappings do not differ significantly from those triggered by native Polish sounds; however, the strength of the native mappings expressed in percentage values appears to be relatively higher.

The strongest colour mappings are generated by high front vowels; English /i:/ and Polish /i/, which are consistently mapped onto yellow and green across the studies. A comparable degree of strength of cross-modal association is demonstrated by Polish /e/, which is prevailingly linked with green and blue; however, its English counterpart generated less strong associations (Study 2 and 3). Open vowels reveal moderately strong tendencies to be mapped onto red and its related hues, with /æ/ triggering red, yellow, orange (Study 2) and red, orange, brown (Study 3); /a:/ being mapped onto red and black (Study 2) and red and brown (Study 3); and Polish/a/ onto red, orange and brown. Grey appears to be an indicator of a central tongue position as it is the colour generated most consistently across the studies by English central vowels, i.e. / / / - grey and white (Study 2 and 3), /3:/ - grey, brown, black (Study 2 and 3). Moreover, fronted back /u/ as well as retracted front $/ \mathrm{I} /$ and Polish /i/ feature grey in their most significant sound-colour combinations. Back vowels, in turn, are consistently associated with dark hues across the studies and across languages. High back English /u:/ is mapped with moderate strength onto a mixture of brown, blue and black (Study 2), and brown and blue (Study 3), while Polish /u/ onto blue and brown. On the other hand, / $/ \mathrm{i} /$ is pre- 
Table 7. A cross-study comparison of vowel-colour mappings for English and Polish. (Study 1 - Wrembel 2007; Study 2 - Wrembel and Rataj 2008; Study 3 - present study.)

\begin{tabular}{|c|c|c|}
\hline \multicolumn{3}{|c|}{ Study 2: English } \\
\hline \multirow[t]{2}{*}{ i: } & yellow & $28 \%$ \\
\hline & green & $20 \%$ \\
\hline I & grey & $17 \%$ \\
\hline \multirow[t]{2}{*}{ e } & red & $19 \%$ \\
\hline & green & $16 \%$ \\
\hline \multirow[t]{3}{*}{$æ$} & red & $21 \%$ \\
\hline & yellow & $17 \%$ \\
\hline & orange & $11 \%$ \\
\hline \multirow[t]{2}{*}{$\Lambda$} & grey & $15 \%$ \\
\hline & green & $14 \%$ \\
\hline \multirow[t]{2}{*}{ a: } & red & $16 \%$ \\
\hline & black & $15 \%$ \\
\hline \multirow[t]{2}{*}{$\partial$} & grey & $23 \%$ \\
\hline & white & $13 \%$ \\
\hline \multirow[t]{3}{*}{ 3: } & grey & $20 \%$ \\
\hline & brown & $19 \%$ \\
\hline & black & $16 \%$ \\
\hline $\mathrm{D}$ & $\begin{array}{l}\text { non } \\
\text { signific }\end{array}$ & \\
\hline 0: & brown & $29 \%$ \\
\hline \multirow[t]{2}{*}{$U$} & grey & $28 \%$ \\
\hline & brown & $17 \%$ \\
\hline \multirow[t]{3}{*}{ u: } & brown & $24 \%$ \\
\hline & blue & $13 \%$ \\
\hline & black & $13 \%$ \\
\hline
\end{tabular}

\begin{tabular}{lc}
\hline \multicolumn{2}{l}{ Study 3: English } \\
\hline yellow & $26 \%$ \\
green & $16 \%$ \\
\hline grey & $14 \%$ \\
yellow & $13 \%$ \\
\hline green & $14 \%$ \\
blue & $13 \%$ \\
\hline red & $18 \%$ \\
orange & $12 \%$ \\
brown & $12 \%$ \\
\hline brown & $14 \%$ \\
purple & $14 \%$ \\
\hline red & $20 \%$ \\
brown & $16 \%$ \\
\hline grey & $22 \%$ \\
white & $13 \%$ \\
\hline brown & $18 \%$ \\
black & $16 \%$ \\
grey & $15 \%$ \\
\hline orange & $17 \%$ \\
\hline brown & $18 \%$ \\
blue & $15 \%$ \\
\hline grey & $18 \%$ \\
\hline purple & $13 \%$ \\
brown & $19 \%$ \\
\hline & $15 \%$ \\
\hline
\end{tabular}

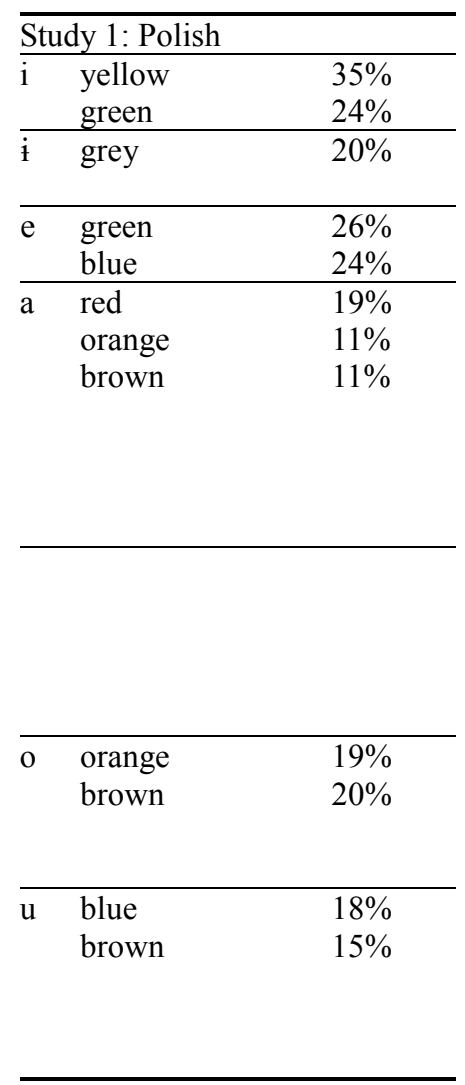

dominantly brown (Study 2) or brown and blue (Study 3); / $/$ / generates orange (Study 3 ) and no significant associations (Study 2), while Polish /o/ is associated mostly with orange and brown. The weakest and least consistent interactions are visible for $/ \Lambda /$, which was associated with grey and green (Study 2) and brown and purple (Study 3).

\section{Discussion}

Addressing the first research question posed at the onset of the study, the analysis of the results reveals that English vowel sounds are mapped on the visual domain of the colour spectrum in a fairly consistent and non-arbitrary manner (see section 3.2.1). This is demonstrated by the internal consistency of the study, i.e. comparable patterns of 
vowel-colour mappings in different conditions for specific vowels and vowel categories (see Tables 2-5) as well as external consistency with the previous studies (see Table 7).

The second question concerned the extent to which sound-colour associations were conditioned by the participants' level of proficiency and established mental representations of English phonemes. The findings reveal no effect of group on the vowel-colour interactions, i.e. the patterns of colour assignments did not depend on the participants' proficiency level (intermediate vs. advanced) nor the presence or absence of previous phonetic training in the English sound system (cf. Section 3.2.3).

The final question deals with the impact of the condition of presentation of the auditory stimuli on the nature of the cross-modal mappings. The reaction time analysis indicates that less proficient learners took longer to assign colours vowels presented in context than more advanced learners. On the whole, the vowels presented in the CVC context generated longer reaction times than vowels presented in isolation (see section 3.2.4).

Interpreting the findings in the framework proposed by Jakobson (1962) for coloured-hearing synaesthesia, it can generally be concluded that the patterns of associations between phonemic features and colour attributes are also valid in normal, i.e. nonsynaesthetic speech perception. Following Jakobson's predictions, the present findings reveal a visible tendency for vocalic compactness (openness) to generate optimal chromacity, i.e. red (/a:/ was consistently mapped onto red and brown whereas /æ/ onto red and orange). Vocalic diffuseness related to tongue raising tended to be consistently associated with attenuated chromacity (yellow/green/blue) particularly when accompanied by the advancement of the tongue (/i:/ in all conditions triggered strong associations with yellow and green, /u:/ demonstrated non-random associations with blue and brown). Achromaticity, in turn, was found to be related to neutral tongue positions as /o/ pointed to a strong correlation with grey, whereas /3:/ yielded significant associations with grey, brown and black. Moreover, achromatic grey was also associated with centralised tongue positions in the case of $/ \mathrm{I} /$ and $/ \mathrm{v} /$.

Moreover, a clear correspondence was found between Donegan's use of the concept of vowel colour and the results of the present study. As predicted by Donegan (1985), palatal, i.e. front, vowels were significantly more frequently associated with bright colours, as opposed to labial, i.e. back, vowels that generated dark colour associations. Vowels which are neither palatal or labial, which are referred to by Donegan as plain or achromatic, were actually mapped onto grey in the experiment. The findings appear to be also partially consistent with Miyahara's et al. (2006) perceptual similarity experiment in the non-random assignment patterns of red to /a/ and yellow to /i/.

The presented analysis relied mostly on local characteristics of formant frequencies as perceptual parameters used to categorise vowel colour. Sounds with more highfrequency energy, such as /ii/, tend to generate bright colour associations in the experiment (yellow, green), while lower-frequency sounds, e.g. /u:/ trigger darker colours (brown, blue and black). 
The present results also corroborate research findings on synaesthesia that reported a tendency among both synaesthates and non-synaesthetes to associate high pitch prominent sounds with lighter colours and low pitch tones with darker colours (Marks 1987; Ward et al. 2006). This may lead to the conclusion that colour-sound associations reported in synaesthesia tend to be non-random also in a normal non-synaesthetic population, as it lends some support for Ward et al.'s (2006) hypothesis that synaesthetes and non-synaesthetes apply the same cognitive mechanisms to map sounds to colours that involve pitch-lightness mappings. Furthermore, the findings offer some support for the cross-modal nature of normal perception. However, the sound-colour associations generated by the general public cannot be expected to equal the consistency and specificity of colours demonstrated by coloured-hearing synaesthetes, as shown also by Ward at al. (2006) and Simner et al. (2005).

\section{Pedagogical implications}

The results of this and the present author's previous studies point to the possibility of strategically-generated visual imagery (i.e. colours) that may accompany and reinforce conscious perceptual experience of speech sounds and thus be applicable to the process of foreign language teaching to facilitate the acquisition of foreign language sounds. There is evidence that by combining information from different senses we can enhance stimulus detection and that multi-sensory interactions can influence conscious perception of the properties of stimuli (Watkins et al. 2006).

Therefore, the expected outcome of the present investigation is to develop a system of mapping perceptual attributes between audio and visual senses and thus to propose a colour-coded vowel chart that would function as a pedagogical aid based on the idea of multimodal reinforcement techniques. The application of the coloured vowel chart would involve better visualisation, reinforcement and practice of fine phonemic distinctions. Colour-coding could also be applied to facilitate the use of transcription symbols and to illustrate spelling-sound correspondence with graphemes being coloured in accordance with the master sound chart. A preliminary proposal for such a coloured vowel chart was presented in Wrembel (2007).

\section{Conclusions}

The vowel-colour interactions generated in the present study demonstrate non-arbitrary patterns of distribution, in which specific phonemic features trigger particular colour attributes, namely front vowels are associated with bright colours; back vowels with darker hues; open vowels generally with red; and central vowels with achromatic grey. This may serve as an experimental validation of most of the predictions for vowel colours put forward by Jakobson (1962) and Donegan (1985). 
The series of studies by the present author provide support for natural and quite stable tendencies in sound-colour associations, as they demonstrate consistency over time, across the studies and for different L2 proficiency levels (cf. Wrembel 2007; Wrembel and Rataj 2008; present study). Furthermore, judging by the similarities between L1 and L2 vowel-colour mappings, the nature of these associations appears to be crosslinguistic, yet it remains to be investigated whether there are any language-specific regularities as well.

Moreover, the results of the present study corroborate the hypothesis that also in non-synaesthetic perception there may exist associations between speech sounds and colours because of similar mechanisms of cross-modal perception, as previously indicated by Ward et al. (2006). This, in turn, may lend further support to the concept of weak synaesthesia (Martino and Marks 2001), which emphasises cross-sensory correspondences expressed through language and perceptual interactions. Finally, colourcoded phonemes may be hypothesized to reflect some universal tendencies in sound symbolism.

Further systematic research into the phenomenon of sound-colour mappings is definitely needed to verify this hypothesis more thoroughly. The areas for future research involve a modified visual stimuli presentation mode (i.e. an application of a more psychologically plausible representation of colours based on Munsell's colour space), testing native associations for English vowel-colour mappings and assuming a wider crosslinguistic perspective.

\section{REFERENCES}

Berlin, B. and P. Kay. 1969. Basic colour terms: Their universality and evolution. Berkeley, CA: University of California Press.

Calvert, G.A. 2001. "Crossmodal processing in the human brain: Insights from functional neuroimaging studies". Cerebral Cortex 11. 1110-1123.

Dailey, A., C. Martindale and J. Borkum. 1997. "Creativity, synaesthesia, and physiognomic perception". Creativity Research Journal 10. 1-8.

Day, S. 2004. Trends in synesthetically coloured graphemes and phonemes. $<$ http://home.comcast.net/ sean.day/Trends2004.htm> Last accessed: 5 Jan 2007.

Donegan, P. 1985. On the Natural Phonology of vowels. New York: Garland Publishing.

Flagg, L. and J. Stewart. 1985. "Studying speech perception in adolescent school-age children by utilizing primary colour perception”. Journal of Psycholinguistic Research 14. 67-80.

Harrison, J. 2001. Synaesthesia: The strangest thing. Oxford: Oxford University Press.

Hinton, L., J. Nichols and J. Ohala. 1994. Sound symbolism. Cambridge: Cambridge University Press.

Hubbard, T.L. 1996. "Synesthesia-like mappings of lightness, pitch and melodic interval". American Journal of Psychology 109. 219-238.

Jakobson, R. 1962. Selected writings: I Phonological Studies. The Hague: Mouton.

Klunder, K. 2003. Auditory color constancy.

$<$ http://www.acoustic.org/press/146th/Kluender.htm> Last accessed: 20 Dec 2006. 
Lyons, A. 2001. "Synaesthesia - A cognitive model of cross-modal association". Consciousness, Literature and the Arts 2(2). 1-10.

Marks, L. 1975. "On coloured-hearing synaesthesia: Cross-modal translations of sensory dimensions". Psychological Bulletin 82(3). 303-331.

Martino, G. and L.E. Marks. 2001. "Synesthesia: Strong and weak". Current Directions in Psychological Science 10. 61-65.

Miyahara, T., T. Amemiya and R. Sekiguchi. 2006. "A psychological experiment on nonsynesthetes correspondence between colours and voiced vowels". Proceedings of the First International Workshop in Kansei, Japan. 102-105.

Simner, J., J. Ward, A. Jansari, K. Noonan and D. Oakley. 2005. "Non-random associations of graphemes to colours in synaesthetic and non-synaesthetic populations". Cognitive Neuropsychology 22. 1-17.

Slawson, W. 1985. Sound colour. University of California Press.

Ward, J., B. Huckstep and E. Tsakanikos. 2006. "Sound-colour synaesthesia: To what extent does it use cross-modal mechanisms common to us all?” Cortex 42. 264-280.

Watkins, S., L. Shams, S. Tanaka, J. Haynes and G. Rees. 2006. "Sound alters activity in human V1 in association with illusory visual perception". NeuroImage 31. 1247-1256.

Wrembel, M. 2007. "Still sounds like a rainbow - A proposal for a coloured vowel chart". Proceedings of the Phonetics Teaching and Learning Conference (PTLC2007), London. 1-4.

Wrembel, M. and K. Rataj. 2008. "Sounds like a rainbow - Sound-colour mappings in vowel perception". Proceedings of the 2nd ISCA Workshop on Experimental Linguistics (ExLing 2008), Athens. 237-240.

\section{Address correspondence to:}

Magdalena Wrembel

School of English

Adam Mickiewicz University

Al. Niepodległości 4

61-874 Poznań

Poland

magdala@amu.edu.pl 